Cite this: Polym. Chem., 2014, 5, 3817

\title{
Aminobenzodione-based polymers with low bandgaps and solvatochromic behavior $\dagger$
}

\author{
Haichang Zhang, ${ }^{a}$ Saman Ghasimi, ${ }^{a}$ Bernd Tieke, ${ }^{* a}$ Alexander Schade ${ }^{b}$ \\ and Stefan Spange ${ }^{b}$ \\ $\mathrm{Pd}$-catalyzed amination polymerization of deep blue aminobenzodifuranone monomer 3-(4-bromo-phenyl)- \\ 7-(4-octylaminophenyl)-benzo[1,2-b:4,5- $\left.b^{\prime}\right]$-difuran-2,6-dione (M1) is described, as well as polymerization of \\ symmetric dibromophenyl-benzodifuranone (M2), or the corresponding dibromophenylbenzodipyrrolidone \\ (M3) with $N, N^{\prime}$-dialkylated phenylenediamines (M4a,b). The resulting polymers P1-3 exhibit low bandgaps \\ (1.08-1.47 eV), broad UV/vis absorption bands (400-900 nm), and a large solvatochromic shift up to \\ $3140 \mathrm{~cm}^{-1}$ from $n$-hexane to hexamethylphosphoramide. Multiple linear regression analyses of $\tilde{\nu}_{\max }$ of the \\ solvent-dependent solvatochromic UV/vis absorption bands of M1, P1 and P2a are presented, from which \\ Kamlet-Taft and Catalán solvent parameters were determined. All monomers and polymers exhibit high \\ extinction coefficients up to $8.6 \times 10^{4} \mathrm{~L} \mathrm{~mol}^{-1} \mathrm{~cm}^{-1}$ and high photostability, and might be suitable for \\ electronic applications. \\ Received 11th December 2013 \\ Accepted 16th February 2014 \\ DOI: $10.1039 / c 3 p y 01702 \mathrm{~h}$ \\ www.rsc.org/polymers
}

\section{Experimental section}

\section{Introduction}

Molecular D-A (donor-acceptor) systems are of current interest owing to their potential applications in molecular electronic devices, ${ }^{1}$ nonlinear optics, ${ }^{2}$ and artificial photosynthetic systems. ${ }^{3}$ Among all kinds of electron acceptor moieties, benzodifuranone (BDF), benzodipyrrolidone (BDP) and their derivatives because of their quinonoid structure have received much attention recently as promising candidates for electron-deficient materials. ${ }^{4,5}$ BDF and BDP are high-performance pigments serving as important building blocks for near-infrared (NIR) absorption. They were first developed in the mid-1970s and commercialized as disperse dyes owing to their deep color and high photochemical stability. ${ }^{6}$ Depending on the substitution pattern, BDFs exhibit red to blue colors. $^{7}$

Monomeric aminobenzodifuranone (ABDF) is a deep blue colored dye with interesting solvatochromic behavior, ${ }^{8}$ which has attracted our attention. The aminobenzodifuranone chromophore is a typical D-A system, in which aniline as an electron donor is combined with benzodifuranone as an acceptor unit. To our knowledge, only a few articles reported on derivatives of $\mathrm{ABDF},{ }^{8}$ while polymers based on ABDF are completely unknown.

\footnotetext{
${ }^{a}$ Department of Chemistry, University of Cologne, Luxemburger Str.116, D-50939 Cologne, Germany. E-mail: tieke@uni-koeln.de

${ }^{b}$ Department of Polymer Chemistry, Chemnitz University of Technology, Strasse der Nationen 62, D-09111 Chemnitz, Germany

$\dagger$ Electronic supplementary information (ESI) available: Experimental details, the ${ }^{1} \mathrm{H}$ NMR spectrum of 4-(octyl amino)phenyl tartronic acid, UV/vis absorption spectra of $\mathbf{M} 2$ and $\mathbf{M} 3$ and polymers P1, P2a and P3, before and after irradiation in toluene, and solvatochromic data. See DOI: 10.1039/c3py01702h
}

\section{Materials}

Reagents. 4-Bromo-mandelic acid, alloxan monohydrate, hydroquinone, $\mathrm{N}$-octylaniline, potassium hydroxide, ammonium persulphate, potassium persulfate, $\mathrm{Pd}_{2}(\mathrm{dba})_{3}, \mathrm{X}-\mathrm{Phos}$, cesium carbonate, $t$-BuOK, $n$-octylamine, $n$-2-octyldodecylamine, bromo-2-octyldodecylamine and 1,4-cyclohexanedione were obtained from Aldrich and Fluka and used without further purification. Solvents were of analytical grade and used without further purification. $\mathbf{M} 2,{ }^{4 a} \mathbf{M} 3^{5 a}$ and $\mathbf{M 4 a}, \mathbf{b}^{9}$ were prepared according to literature methods.

4-(Octyl-amino)phenyl tartronic acid. Alloxan monohydrate (1.6 g, $10 \mathrm{mmol}$ ) and $N$-octylaniline $(2.05 \mathrm{~g}, 10 \mathrm{mmol})$ were dissolved in a mixture of $18 \mathrm{ml}$ ethanol, $3 \mathrm{ml}$ methanol and $5 \mathrm{ml}$ water. While stirring, the clear yellowish solution was neutralized with acetic acid ( $1 \mathrm{ml}, 17 \mathrm{mmol})$. Stirring was continued at $60{ }^{\circ} \mathrm{C}$ for 5 hours. After the reaction, the solvent was removed upon vacuum evaporation. The raw mixture was dissolved in a small amount of ethanol and precipitated in water. A fawn flaky substance was obtained after decantation and drying, with 54\% (1.86 g) yield of 5-hydroxy-5-(4-octylaminophenyl)-barbituric acid. $1.86 \mathrm{~g}$ ( $5.4 \mathrm{mmol})$ of this intermediate were dissolved in an aqueous potassium hydroxide solution ( $3.1 \mathrm{~g}, 55 \mathrm{mmol}, 1.4 \mathrm{M}$ ) and stirred between 40 and $45^{\circ} \mathrm{C}$ for 5 hours. The solution was acidified with acetic acid and water was added. The product precipitated overnight. After filtration and drying, $0.78 \mathrm{~g}$ (yield $40 \%$ ) of a light brown powder were obtained, which showed high purity by NMR analysis. ${ }^{1} \mathrm{H}$ NMR (300 MHz, $\mathrm{d}_{6}$-DMSO) $\delta$ ppm: 7.03-7.06 (d, 2H), 6.55-6.58 (d, 2H), 6.27-6.28, (d, 1H), $5.83(\mathrm{~s}, 1 \mathrm{H}), 2.98(\mathrm{~s}, 2 \mathrm{H}), 1.50-1.52(\mathrm{t}, 2 \mathrm{H}), 1.26(\mathrm{~s}, 10 \mathrm{H}), 0.86$ 
(t, 3H). ${ }^{13} \mathrm{C}$ NMR (300 MHz, d 6 -DMSO) $\delta$ ppm: 172.9, 154.9, 149.9, 128.3, 118.9, 112.7, 82.8, 43.7, 29.4, 29.2, 27.1, 22.7, 14.1. 3-(4-Bromophenyl)-7-(4-octylaminophenyl)-benzo[1,2-b:4,5$b^{\prime}$ ]-difuran-2,6-dione (M1). 5-Hydroxy-2-oxo-3-(4-bromophenyl)-2,3-dihydrobenzo[1,2- $b$ ]furan $(1.39 \mathrm{~g}, 4.6 \mathrm{mmol})$ and 4-(octyl-amino)phenyl tartronic acid mono potassium salt $(2.48 \mathrm{~g}, 7.2 \mathrm{mmol})$ were stirred in acetic acid $(20 \mathrm{ml})$. The mixture was heated to $110{ }^{\circ} \mathrm{C}$ and stirred for $5 \mathrm{~h}$ at this temperature. $1.05 \mathrm{~g}(4.6 \mathrm{~mol})$ of ammonium persulphate was added in 8 portions at $80^{\circ} \mathrm{C}$. The mixture was stirred at $95{ }^{\circ} \mathrm{C}$ for $1 \mathrm{~h}$. Subsequently, the mixture was cooled to room temperature and a small amount of water was added. The precipitate was filtered, washed with a small amount of acetic acid, and then with water. The crude product was refluxed in methanol giving a dark solid (1.05 g, yield: $42 \%) .{ }^{1} \mathrm{H}$ NMR $\left(300 \mathrm{MHz}, \mathrm{CDCl}_{3}\right) \delta \mathrm{ppm}: 7.83-7.96(\mathrm{~d}, 2 \mathrm{H}), 7.65-7.72(\mathrm{~m}$, $4 \mathrm{H}), 6.99(\mathrm{~s}, 1 \mathrm{H}), 6.82(\mathrm{~s}, 1 \mathrm{H}), 6.69-6.72(\mathrm{~d}, 2 \mathrm{H}), 4.37(\mathrm{~s}, 1 \mathrm{H})$, 3.22-3.27 (t, 2H), 1.64-1.72 (m, 2H), $1.32(\mathrm{~s}, 10 \mathrm{H}), 0.89-0.95$ $(\mathrm{t}, 3 \mathrm{H}) .{ }^{13} \mathrm{C}$ NMR could not be measured. UV/vis (DCM): 628 $\mathrm{nm} ; \varepsilon(628) / \mathrm{L} \mathrm{mol}^{-1} \mathrm{~cm}^{-1}$ : 65241.

Polymer P1. In a Schlenk flask, M1 (300 mg, $0.55 \mathrm{mmol})$, potassium phosphate $(584 \mathrm{mg}, 2.75 \mathrm{mmol}), \mathrm{Pd}_{2}(\mathrm{dba})_{3}(15.1$ $\mathrm{mg}, 0.016 \mathrm{mmol}$ ) and X-Phos $(50 \mathrm{mg}, 0.11 \mathrm{mmol})$ are dissolved in dioxane $(6 \mathrm{ml})$ under nitrogen. The mixture is heated to $100{ }^{\circ} \mathrm{C}$ and stirred for 40 hours under nitrogen. After cooling to room temperature, water is added in order to precipitate the product. The precipitate is filtered off and washed with methanol. After extraction with toluene, a black solid is obtained (95 mg, yield: $37 \%) .{ }^{1} \mathrm{H}$ NMR $(300 \mathrm{MHz}$, $\left.\mathrm{CDCl}_{3}\right) \delta$ ppm: 7.74-7.80 (m, 4H), 7.33-7.61 (m, 4H), 6.72 $(\mathrm{s}, 2 \mathrm{H}), 3.08-6.22(\mathrm{~s}, 2 \mathrm{H}), 1.51-1.72(\mathrm{~s}, 2 \mathrm{H}), 1.06-1.40$ (m, 10H), 0.77-0.95 (t, 3H). Molecular weight (GPC, THF): $M_{\mathrm{w}}=5.9 \mathrm{kDa}, \mathrm{PD}=2.2$. UV/vis (DCM): $645 \mathrm{~nm}, \mathrm{UV} / \mathrm{Vis}$ (thin film): $708 \mathrm{~nm} ; \varepsilon(645) / \mathrm{L} \mathrm{mol}^{-1} \mathrm{~cm}^{-1}$ : 30600 .

Polymer P2a. In a Schlenk flask, M2 $(214 \mathrm{mg}, 0.43 \mathrm{mmol})$, $N, N$-bis(2-ethylhexyl)benzene-1,4-diamine dihydrochloride salt (M4a) (175 mg, $0.43 \mathrm{mmol}), \mathrm{Pd}_{2}(\mathrm{dba})_{3}$ (11.9 mg, $\left.0.015 \mathrm{mmol}\right)$ and X-Phos (39.2 mg, $0.082 \mathrm{mmol}$ ) are dissolved in a mixture of DMF-toluene $(2: 1)(6 \mathrm{ml})$ under nitrogen. Then, cesium carbonate $(310 \mathrm{mg}, 0.95 \mathrm{mmol})$ is added and the mixture is allowed to stir for 48 hours under nitrogen at $90{ }^{\circ} \mathrm{C}$. After completion of the reaction, the dark blue solution is treated with DCM, washed three times with water and once with brine. Then the organic layer is dried over anhydrous magnesium sulfate, and the solvent is removed at reduced pressure. Subsequently the crude product is dissolved in a minimal amount of DCM and precipitated in methanol. The product is obtained as a dark solid (181.2 mg, yield: 63\%). ${ }^{1} \mathrm{H}$ NMR (300

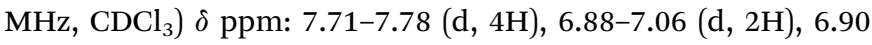
$(\mathrm{s}, 4 \mathrm{H}), 6.71-6.78(\mathrm{~d}, 2 \mathrm{H}), 6.63-6.69(\mathrm{~d}, 2 \mathrm{H}), 3.55-3.65(\mathrm{~d}, 2 \mathrm{H})$, 3.03-3.09 (d, 2H), 1.70-1.86 (br, 2H), 1.22-1.49 (m, 16H), 1.06$1.40(\mathrm{~m}, 10 \mathrm{H}), 0.88-0.99(\mathrm{~m}, 12 \mathrm{H})$. Molecular weight (GPC, THF): $M_{\mathrm{w}}=5.7 \mathrm{kDa}, \mathrm{PD}=1.5$. UV/vis (DCM): $709 \mathrm{~nm}, \mathrm{UV} / \mathrm{vis}$ (thin film): $724 \mathrm{~nm} ; \varepsilon$ (709)/L mol${ }^{-1} \mathrm{~cm}^{-1}: 16721$.

Polymer P2b. In a Schlenk flask, M2 (137.6 mg, $0.28 \mathrm{mmol})$, $N, N$-bis(2-octyldodecyl)benzene-1,4-diamine dihydrochloride salt (M4b) (205 $\mathrm{mg}, 0.28 \mathrm{mmol}), \mathrm{Pd}_{2}(\mathrm{dba})_{3}(7.6 \mathrm{mg}, 0.008$ $\mathrm{mmol}$ ) and X-Phos (25 $\mathrm{mg}, 0.053 \mathrm{mmol})$ are dissolved in a mixture of DMF-toluene (2:1) $(3.2 \mathrm{ml})$ under nitrogen. Then, cesium carbonate $(198 \mathrm{mg}, 0.60 \mathrm{mmol})$ is added and the mixture is allowed to stir for 48 hours under nitrogen at $90{ }^{\circ} \mathrm{C}$. After completion of the reaction, the dark solution is treated with DCM, washed three times with water and once with brine. Then the organic layer is dried over anhydrous magnesium sulfate and the solvent is removed at reduced pressure. Subsequently, the crude product is dissolved in a minimal amount of DCM and precipitated in methanol. The product is obtained as a dark blue solid (156.5 mg, yield: 54\%). ${ }^{1} \mathrm{H}$ NMR (300 MHz, $\mathrm{CDCl}_{3}$ ) $\delta \mathrm{ppm:} \mathrm{7.70-7.76} \mathrm{(d,} \mathrm{4),} 7.28$ (s, 2H), 6.96-7.02 (d, 4H), 6.61-6.66 (d, 2H), 6.51-6.59 (d, 2H), $3.58(\mathrm{~s}, 2 \mathrm{H}), 3.03(\mathrm{~s}, 2 \mathrm{H}), 2.04(\mathrm{~s}, 2 \mathrm{H}), 1.12-1.43(\mathrm{br}, 64 \mathrm{H})$, 0.88-0.93 (br, 12H). Molecular weight (GPC, THF): $M_{\mathrm{w}}=9.7$ $\mathrm{kDa}, \mathrm{PD}=1.7$. UV/vis (DCM): $725 \mathrm{~nm}$, UV/vis (thin film): $741 \mathrm{~nm} ; \varepsilon(725) / \mathrm{L} \mathrm{mol}^{-1} \mathrm{~cm}^{-1}: 23911$.

Polymer P3. In a Schlenk flask, M3 (100.0 mg, $0.095 \mathrm{mmol})$, M4b (38.4 mg, $0.095 \mathrm{mmol}), \mathrm{Pd}_{2}(\mathrm{dba})_{3}(2.6 \mathrm{mg}, 0.029 \mathrm{mmol})$ and X-Phos $(8.2 \mathrm{mg}, 0.017 \mathrm{mmol})$ are dissolved in dry toluene $(3 \mathrm{ml})$ under nitrogen. Then, $t$-BuOK ( $45 \mathrm{mg}, 0.44 \mathrm{mmol}$ ) is added and the mixture is allowed to stir for 24 hours under nitrogen at $90{ }^{\circ} \mathrm{C}$. After completion of the reaction, the dark blue solution is treated with DCM, washed three times with water and once with brine. Then the organic layer is dried over anhydrous magnesium sulfate and the solvent is removed at reduced pressure. After that, the crude product is dissolved in a minimal amount of DCM and precipitated in methanol. The product is obtained as a dark blue solid (65.7 mg, yield: 57\%). 7.59-7.75 (br, 4H), 7.17 (s, 2H), 6.70$6.77(\mathrm{~d}, 4 \mathrm{H}), 6.71-6.78$ (d, 2H), 6.60-6.68 (d, 2H), 3.46-3.03 (br, 6H), 3.01-3.10 (br, 2H), 1.83 (s, 4H), 1.11-1.47 (br, 80H), 0.79-1.01 (br, 24H). Molecular weight (GPC, THF): $M_{\mathrm{w}}=10.1 \mathrm{kDa}$, $\mathrm{PD}=1.5$. UV/vis (DCM): $623 \mathrm{~nm}$, UV/vis (thin film): $638 \mathrm{~nm}$; $\varepsilon(623) / \mathrm{L} \mathrm{mol}^{-1} \mathrm{~cm}^{-1}: 26762$.

\section{Methods}

Instrumentation. UV/vis absorption spectra were recorded using a Perkin-Elmer Lambda 14 spectrometer. Photoluminescence spectra were recorded using a Perkin-Elmer LS50B spectrometer. The photostability was recorded using a $200 \mathrm{~W} \mathrm{Hg}$ lamp (Oriel Instruments 6283) at a distance of $20 \mathrm{~cm}$ at room temperature. ${ }^{1} \mathrm{H}$ NMR spectra were recorded using a Bruker DPX 300 spectrometer, which operates at $300 \mathrm{MHz}$. Molecular weights were determined by size exclusion chromatography (SEC) using a Water/Millipore UV detector 481 and a mixed gel column (Latek/Styragel 50/1000 nm pore size). All measurements were carried out in tetrahydrofuran at $45{ }^{\circ} \mathrm{C}$. The column was calibrated using commercially available polystyrene standards. Cyclic voltammograms were recorded using a potentiostat PG 390 from Heka Company. The thin films of the polymers were cast on an ITO electrode and cycled in acetonitrile (saturated with nitrogen) containing $0.1 \mathrm{M}$ tetrabutylammonium hexafluorophosphate $\left(\mathrm{TBAPF}_{6}\right)$ as the electrolyte salt. Platinum was used as reference and counter electrodes. The voltage data were calculated for the ferrocene/ferrocenium redox couple. The scan rate was $100 \mathrm{mV} \mathrm{s}^{-1}$ and the temperature was $20^{\circ} \mathrm{C}$. 


\section{Results and discussion}

In this article, we report on the novel monomer M1 and four aminobenzodione-based $\pi$-conjugated polymers. M1 was prepared according to Scheme 1 which is very soluble in common organic solvents and suitable for Buchwald-Hartwig amination and Stille, Heck and Suzuki coupling because of the bromine and alkylamino end groups.

The polymers consist of amino groups as electron-donating units, and BDF or BDP as electron-accepting units causing low bandgaps, broad UV/vis absorption and high photostability. BDF/BDP-based monomers $\mathbf{M} 2$ and $\mathbf{M} 3$, and the $N, N^{\prime}$-dialkylaminobenzene monomer $\mathbf{M 4}$ were prepared according to the literature. ${ }^{4 a, 5 a, 9}$ The polymers were synthesized by BuchwaldHartwig amination using $\operatorname{Pd}_{2}(\mathrm{dba})_{3}$ as the catalyst and X-Phos as the ligand (Scheme 2). ${ }^{10}$
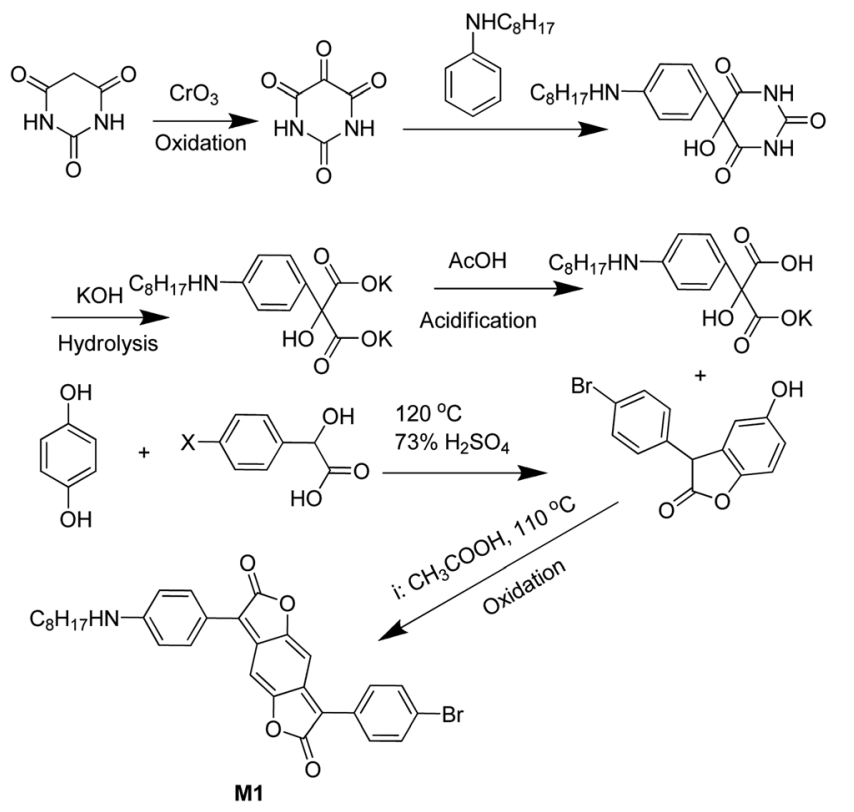

Scheme 1 Synthesis and structure of M1.
The polymers were characterized by ${ }^{1} \mathrm{H}$ NMR spectroscopy (Fig. 1) and gel permeation chromatography (GPC). The signals around 0.8-3.3 ppm originate from the alkyl groups, while the signals around 6.72-7.96 are typical for the protons of the core of $\mathrm{BDF}$ and the phenyl groups attached to the core. For M1, the chemical shifts at $4.37 \mathrm{ppm}$ originate from the protons attached to the amino group while these signals disappear for the polymers due to polymerization. The number-average molecular weights were found to be 5.9 kDa (PDI: 2.2, P1), 5.7 kDa (PDI: 1.5, P2a), 9.7 kDa (PDI: 1.7, P2b) and 10.1 kDa (PDI: 1.5, P3), respectively.

\section{Optical properties}

In Fig. 2, the UV/vis spectrum of M1 in dichloromethane solution is shown. M1 exhibits a broad UV/vis absorption band with a maximum at $628 \mathrm{~nm}$, the extinction coefficient of the maximum being $6.5 \times 10^{4} \mathrm{~L} \mathrm{~mol}^{-1} \mathrm{~cm}^{-1}$. The maximum of M1 is $121 \mathrm{~nm}$ red-shifted compared with $\mathbf{M} 2$ in dichloromethane since the alkylamino unit is a powerful donor, which shifts the UV/vis absorption to a longer wavelength. M1 is readily soluble in common organic solvents such as toluene, $\mathrm{N}, \mathrm{N}$-dimethylformamide and so on, in contrast to $\mathbf{M} 2$, which is not very soluble in toluene or dichloromethane, and therefore not suitable for Suzuki coupling. This is firstly due to the presence of the amino group, which can form a highly polar resonance structure and interact with adjacent solvent molecules (see a suggested mechanism in S4 $\dagger$ ), and secondly the alkyl substituent of the amino group favors the solubility.

In Fig. 3, the UV/vis absorption spectra of the polymers in dichloromethane solution and as thin films are shown. All polymers are deep colored, the extinction coefficients of the strongest bands being 1.7-3.1 $\times 10^{4} \mathrm{~L} \mathrm{~mol}^{-1} \mathrm{~cm}^{-1}$. The $\mathrm{UV} /$ vis absorption spectra of P2a and P2b in dichloromethane exhibit broad bands with maxima at 709 and $724 \mathrm{~nm}$. The maximum of P2b is slightly red-shifted compared with P2a, since $\mathbf{P 2 b}$ has a higher molecular weight and a more strongly extended $\pi$-system. The UV/vis absorption spectrum of P3 in dichloromethane exhibits a strong maximum at $623 \mathrm{~nm}$. Compared with P2a and P2b, the maximum of P1 is blue-

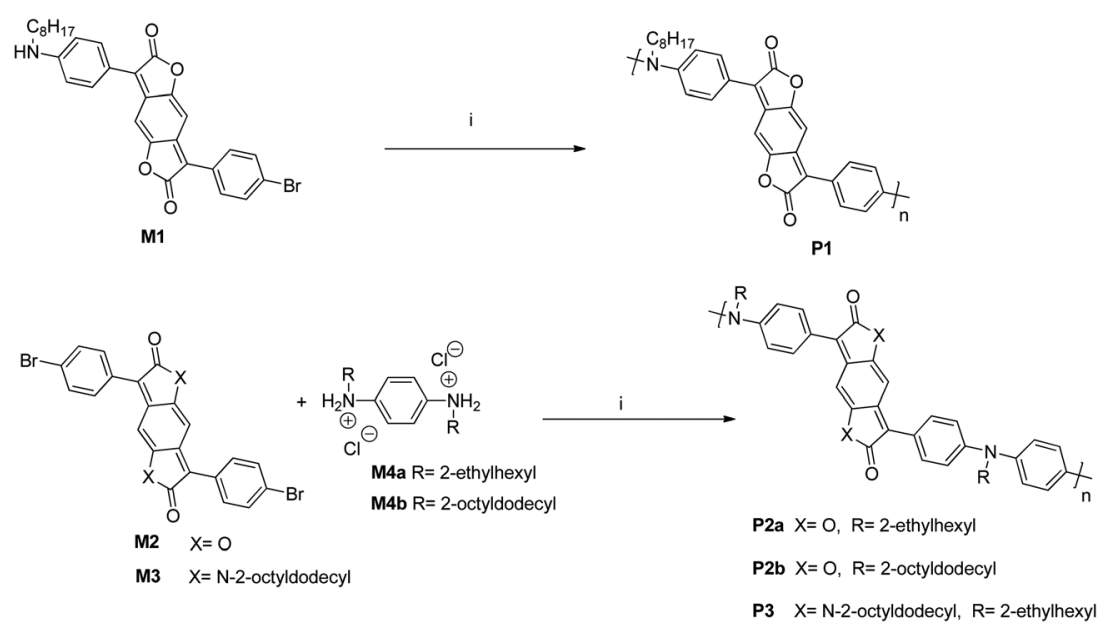

Scheme 2 Synthesis and structure of polymers P1-3. (i) $\mathrm{Pd}_{2}(\mathrm{dba})_{3}, \mathrm{X}-\mathrm{Phos}, \mathrm{Cs}_{2} \mathrm{CO}_{3}$ (t-BuOK for P3), DMF-toluene (2/1) (dioxane for P1 and P3), $\mathrm{N}_{2}$. 


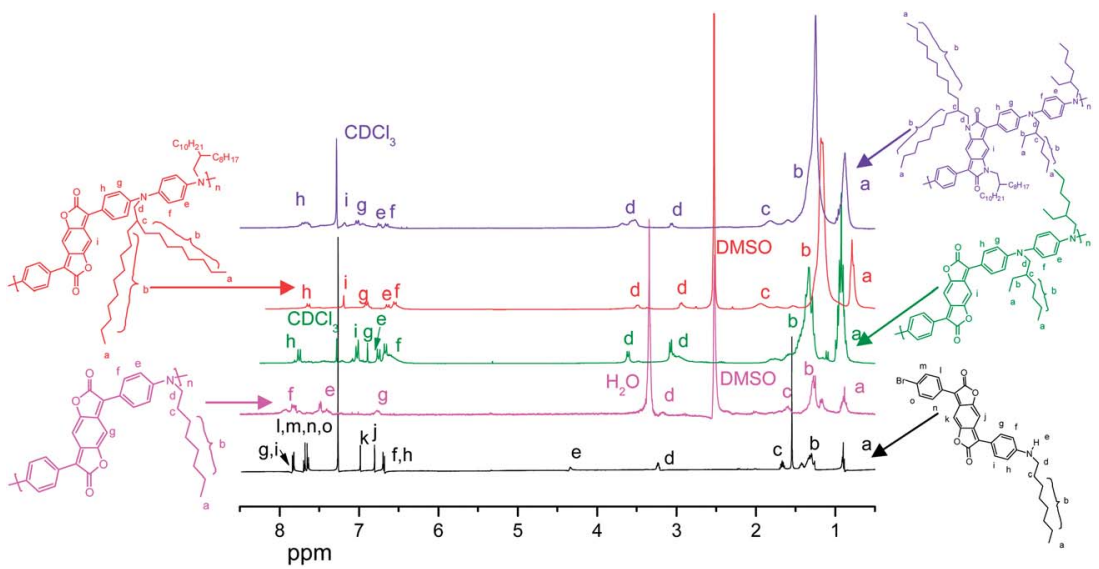

Fig. $1{ }^{1}$ NMR spectra of M1 and P1-P3.

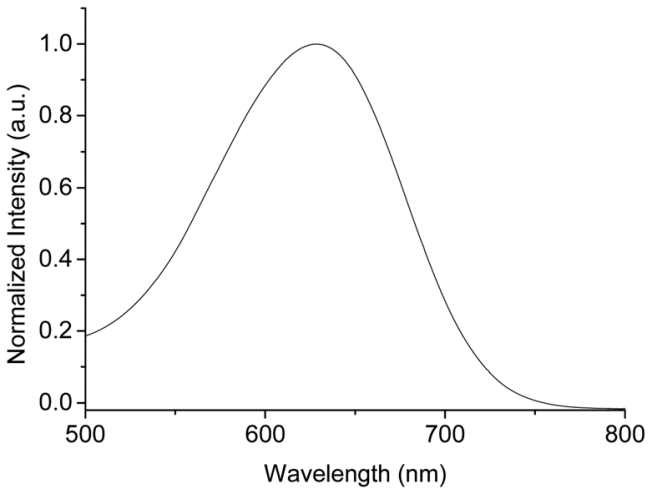

Fig. 2 UV/vis absorption spectra of M1 in dichloromethane.

shifted $(645 \mathrm{~nm})$, giving rise to weaker D-A interactions between the alkylamino groups and the benzodifuranone core. In P2a and P2b enhanced intramolecular charge transfer (ICT) is due to the presence of the $N, N^{\prime}$-dialkyl-1,4phenylenediamine unit with a more powerful donor ability favoring the red shifted absorption. The red shift is stronger than those of the polymers containing the thienyl-benzodione chromophore (88 nm for P1, $152 \mathrm{~nm}$ for P2a, $167 \mathrm{~nm}$ for $\mathbf{P 2 b}$ and $58 \mathrm{~nm}$ for $\mathbf{P 3}$ in dichloromethane, respectively) since the alkylamino group is a more powerful electron donor than the thienyl group. ${ }^{4 a, 5 a}$ All polymers show a very broad
UV/vis absorption from 400 to $900 \mathrm{~nm}$, which matches the solar photon most intense flux (that is in the $400-800 \mathrm{~nm}$ range $)^{11}$ in thin films with maxima between 638 and $725 \mathrm{~nm}$ (Fig. 2 and Table 1). All UV/vis absorption spectra of films are red shifted when compared to dichloromethane solution spectra. The strongest red-shift was found for P1 with $63 \mathrm{~nm}$. This indicates a gain of planar conformation and/or the presence of $\pi-\pi$ interchain association in the solid state. The optical HOMO-LUMO energy gaps estimated from the onset of absorption for films are 1.19-1.47 eV.

Table 1 Optical, band gap and photostability data of the polymers

\begin{tabular}{|c|c|c|c|c|c|}
\hline \multirow[b]{2}{*}{ Polymers } & \multicolumn{2}{|c|}{$\lambda_{\max }[\mathrm{nm}]$} & \multirow[b]{2}{*}{$\begin{array}{l}\text { Extinct. coeff. } \varepsilon\left(\lambda_{\max }\right) \\
{\left[\mathrm{L} \mathrm{mol}^{-1} \mathrm{~cm}^{-1}\right]}\end{array}$} & \multirow[b]{2}{*}{$\begin{array}{l}E_{\mathrm{g}}^{\mathrm{opt} b} / E_{\mathrm{g}}^{\mathrm{ec} c} \\
{[\mathrm{eV}]}\end{array}$} & \multirow[b]{2}{*}{$k^{a}\left[\mathrm{~h}^{-1}\right]$} \\
\hline & $\begin{array}{l}\text { In } \\
\text { DCM }\end{array}$ & $\begin{array}{l}\text { As thin } \\
\text { films }\end{array}$ & & & \\
\hline P1 & 645 & 708 & 30600 & $1.19 / 1.19$ & 0.11 \\
\hline P2a & 709 & 724 & 16721 & $1.31 / 1.23$ & 0.26 \\
\hline P2b & 725 & 741 & 23911 & $1.20 / 1.08$ & 0.33 \\
\hline P3 & 623 & 638 & 26762 & $1.47 / 1.45$ & 0.68 \\
\hline
\end{tabular}

${ }^{a}$ Photostability was determined upon irradiation with a $200 \mathrm{~W} \mathrm{Hg}$ lamp. ${ }^{b}$ Optical bandgap $E_{\mathrm{g}}^{\text {opt }}$ was measured at the onset of absorption of the polymer film $\left(E_{\mathrm{g}}^{\mathrm{opt}}=1240 / \lambda_{\mathrm{abs}, \text { onset }} \mathrm{eV}\right) .{ }^{c} E_{\mathrm{g}}^{\mathrm{ec}}=E_{\mathrm{HOMO}}-E_{\mathrm{LUMO}}$, $-E_{\mathrm{LUMO}}=E_{\text {onset(red) }}+4.8 \mathrm{eV}$ and $-E_{\mathrm{HOMO}}=E_{\text {onset(ox) }}+4.8 \mathrm{eV}$, where $E_{\text {onset(red) }}$ and $E_{\text {onset(ox) }}$ are the onset potentials of the oxidation and reduction processes $v s$. ferrocene.
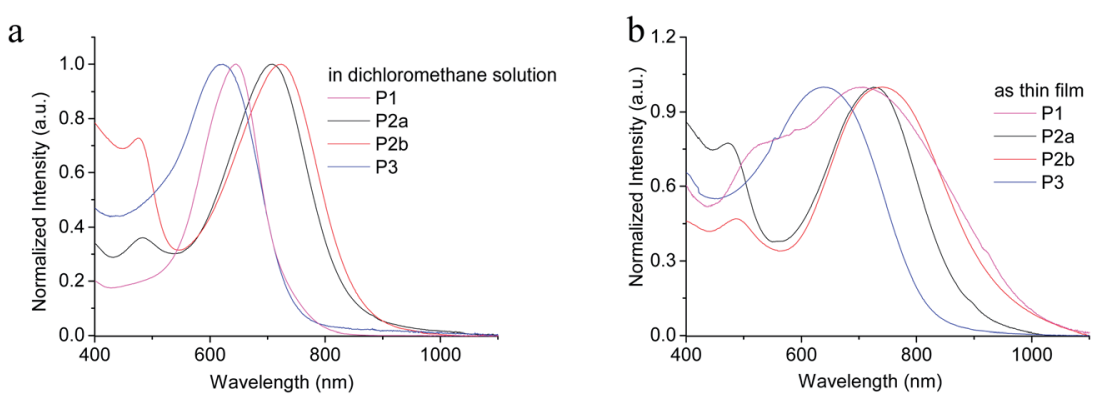

Fig. 3 UV/vis absorption spectra of polymers P1-3 in dichloromethane and as thin films. 


\section{Electrochemical properties}

The electrochemical properties of the four polymers were investigated by cyclic voltammetry. The conditions of the measurement are described in the Experimental part. The HOMO and LUMO energy levels of the polymers were estimated from the onset of the oxidation and reduction curves, respectively (see Fig. 4). It can be seen that anodic oxidation of polymers sets in at low potentials of $0.32 \mathrm{~V}(\mathbf{P 1}), 0.17 \mathrm{~V}$ (P2a), 0.15 V (P2b) and 0.89 V (P3), respectively. Two to three anodic waves with maxima between 0.5 and $1.2 \mathrm{~V}$ occur, which can be ascribed to the formation of cation radicals and dications. The reductive cycles of all polymers exhibit two reversible cathodic waves, which originate from the reduction of the quinonoid to a benzoic structure. This can possibly be explained with a stabilizing negative charge of the oxygen atoms in the carbonyl groups of both the lactone groups in the benzodione units.

All polymers show low LUMO $(-3.79$ to $-4.24 \mathrm{eV})$ and HOMO levels $(-4.95$ to $-5.81 \mathrm{eV})$. Due to the low LUMO energy level of the polymers, good electron injection and ambient stabilities of OFET devices can be expected. ${ }^{5 b}$ The polymers exhibit quite low HOMO-LUMO bandgaps (1.08 to $1.45 \mathrm{eV}$ ). In Table 1, optical and bandgap data of the polymers are compiled.

\section{Photostability properties}

The photostability was studied by exposing toluene solutions of the polymers to a $200 \mathrm{~W} \mathrm{Hg-lamp} \mathrm{at} \mathrm{a} \mathrm{distance} \mathrm{of} 20 \mathrm{~cm}$ and measuring the decrease of the optical absorption $v s$. time. In Fig. 5a, UV/vis absorption spectra of P2b in toluene are shown before and after irradiation for different time periods. Corresponding spectra of M2, M3, P1, P2a and P3 are shown in Fig. S2 and S3. $\dagger$ The plots of $\ln \left(A_{t} / A_{0}\right)\left(A_{t, 0}=\right.$ absorbance at time $t$ and time $t=0) v s$. time for irradiation of polymers lead to nearly straight lines. From the initial slope the rate constants $(k)$ of the photoreaction were derived (Fig. 5 and Table 1). As can be seen from Fig. 5, P2a and P2b are more stable than P1 since the $N, N^{\prime}$-dialkyl-1,4-phenylenediamine unit exhibits a more powerful donor ability than the alkylamino unit. Probably the strong donor-acceptor character of these polymers prevents energy transfer from the amino groups to the BDF core and thus contributes to a higher photostability. Compared with P2a, P3 is less stable, which can be ascribed to the fact that the monomer BDP chromophore is less stable than BDF in UV-light (see S2 $\dagger$ ). In general, polyiminobenzodiones are less stable than isoDPPbased conjugated polymers ${ }^{\mathbf{1 2}}$ or 1,10-naphthodifuranonebased polymers, ${ }^{\boldsymbol{4} \boldsymbol{d}, \mathbf{1 3}}$ but more stable than benzodifuranonebased polymers ${ }^{4 d}$ or DPP-14 and DTPP-based polymers. ${ }^{15}$
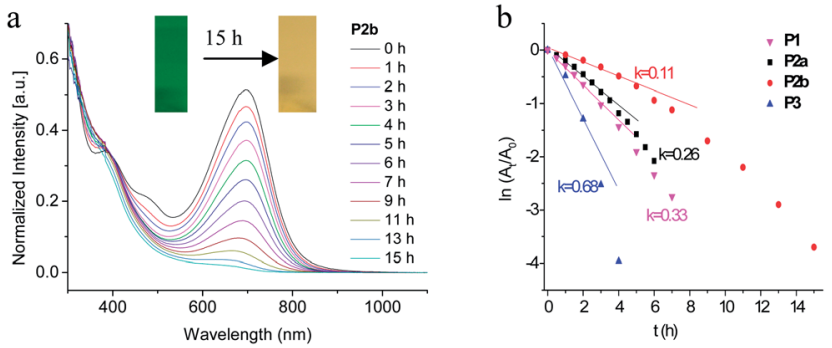

Fig. 5 UV/vis absorption spectra of P2b before and after irradiation in toluene with a $200 \mathrm{~W} \mathrm{Hg}$-lamp and $\ln \left(A_{t} / A_{0}\right) v s$. time for determination of rate constants $(k)$ for polymers.
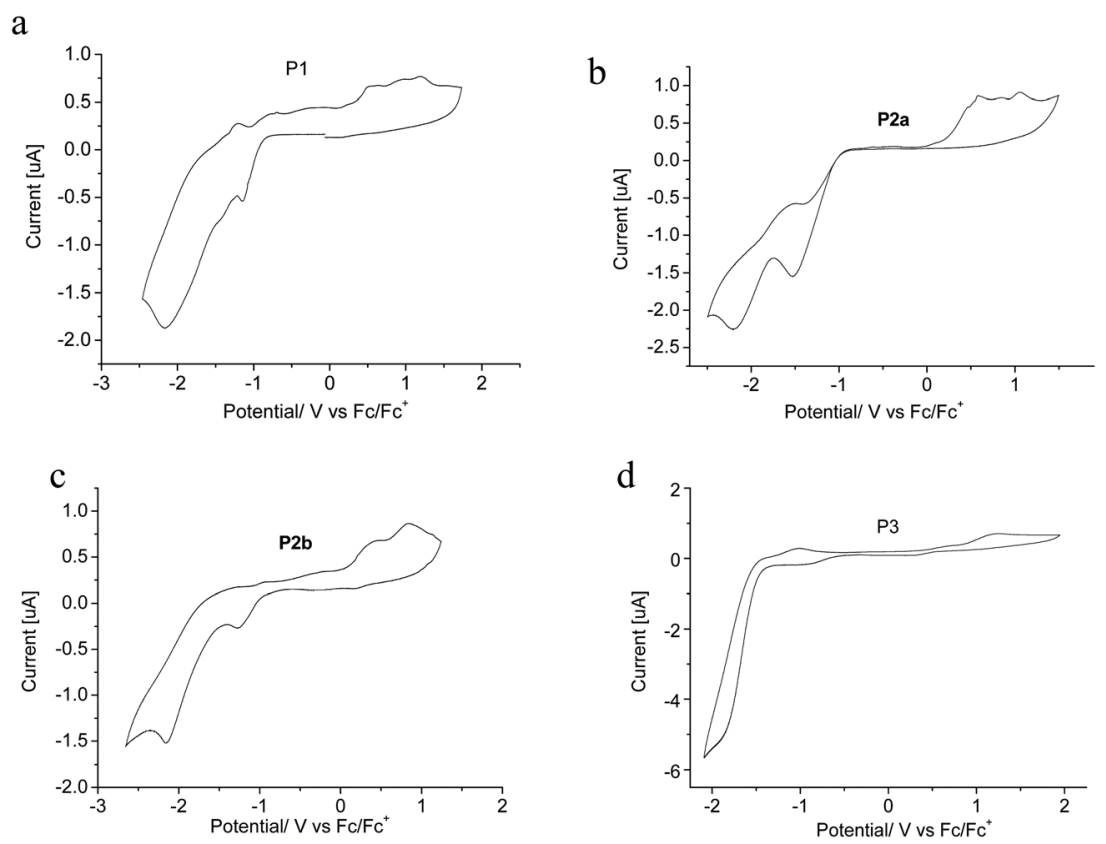

Fig. 4 Cyclic voltammograms of polymers as thin films deposited on the ITO surface. Solution: 0.1 M TBAPF 6 /acetonitrile. Potential calculated versus ferrocene. Scan rate: $100 \mathrm{mV} \mathrm{s}^{-1} ; T=20^{\circ} \mathrm{C}$. 
Table 2 Solvent-independent correlation coefficients $a, b, d$ and e of the Catalán parameters SA, SB, SP and SdP, solute property of the reference system $\tilde{\nu}_{\text {max, }}$ cyclohexane, number of solvents $(n)$, correlation coefficient $(r)$, standard deviation (sd), and significance $(f)$ of the calculated solvatochromism of the model compounds M1, P1 and P2a

\begin{tabular}{|c|c|c|c|c|c|c|c|c|c|}
\hline \multirow[b]{2}{*}{ Compound } & \multicolumn{5}{|c|}{ Catalán equation } & \multicolumn{4}{|c|}{ Correlation data } \\
\hline & $\tilde{\nu}_{\max , 0}$ & $a$ & $b$ & $d$ & $e$ & $n$ & $r$ & sd & $f$ \\
\hline P1 & 20.284 & -0.534 & -1.191 & -5.009 & -1.246 & 33 & 0.93 & 0.284 & $<0.0001$ \\
\hline P2a & 16.629 & 0.137 & -0.428 & -2.452 & -0.541 & 33 & 0.88 & 0.168 & $<0.0001$ \\
\hline
\end{tabular}

\section{Solvatochromic properties}

M1, P1 and P2a were chosen to study the solvatochromic properties. Positive solvatochromic behavior can be attributed to a number of specific and non-specific solute-solvent interactions as summarized in the simplified Kamlet-Taft equation (eqn (1)), ${ }^{16}$ where $s, a$, and $b$ are solvent independent coefficients, and $\pi^{*},{ }^{17 a} \alpha^{17 b}$ and $\beta^{17 c}$ reflect the general solvent dipolarity/polarizability, its specific H-bond donating (HBD) ability, and its specific H-bond accepting (HBA) ability, respectively, which may or may not oppose one another in terms of solvatochromic contributions. $\tilde{\nu}_{\max , 0}$ is the longest wavelength $\mathrm{UV} / \mathrm{vis}$ absorption maximum of the compound measured in a particular solvent where $\pi^{*}=\alpha=\beta=0$ (e.g. cyclohexane) ${ }^{8 a}$

$$
\tilde{\nu}_{\max }=\tilde{\nu}_{\max , 0}+a \alpha+b \beta+s \pi^{*}
$$

Until now, empirical polarity scales have always described the polarizability and dipolarity of the solvent together in one parameter. The first and only successful attempt to separate polarizability and dipolarity was suggested by Catalán with the introduction of solvent acidity (SA), ${ }^{18}$ solvent basicity (SB), ${ }^{19}$ solvent polarizability (SP) ${ }^{20}$ and solvent dipolarity (SdP) $)^{\mathbf{2 1}}$ (eqn (2)).

$$
\tilde{\nu}_{\max }=\tilde{\nu}_{\max , 0}+a \mathrm{SA}+b \mathrm{SB}+d \mathrm{SP}+e \mathrm{SdP}
$$

The three compounds show the shortest wavelength UV/ vis absorption band in $n$-hexane, and exhibit the strongest bathochromic shift in DMSO (for M1, P1 and P2a, $\Delta$ values $\left(\lambda_{\text {max,pol }}-\lambda_{\text {max,nonpol }}\right)$ of $1700 \mathrm{~cm}^{-1}, 2400 \mathrm{~cm}^{-1}$, and 1180 $\mathrm{cm}^{-1}$, respectively). Both equations show good accuracy for M1 with $r>0.9$ (eqn (2) in Table 2 and eqn (1) in Table S2†). It is shown that especially the HBA ability of the solvents $(\beta$ or $\mathrm{SB})$ causes a strong bathochromic shift $(b<0)$. This can be easily explained by $\mathrm{H}$-bonding between the $\mathrm{NH}$-group in the main chain and HBA-solvents. The influence of the $\beta$-term of the solvents on the bathochromic shift of $\mathbf{M 1}$ represents the interactions of HBA capacity solvents with the proton of the $\mathrm{N}-\mathrm{H}$ group, which increases the $+\mathrm{M}$-effect and therefore strengthening of the aromatic push-pull system occurs. HBD-solvents only exhibit a small effect typical for benzodifuranone-based dyes. ${ }^{\boldsymbol{8} \boldsymbol{b}-\boldsymbol{d}}$ A weak bathochromic shift can be recognized due to the interaction with the carbonyl groups $(a<0)$. Polarizability and dipolarity cause a bathochromic shift $\left(\pi^{*}, \mathrm{SP}\right.$ and SdP $\left.<0\right)$, respectively. The solvatochromic range of $\mathbf{M 1}$ is $1700 \mathrm{~cm}^{-1}$ from $n$-hexane to DMSO.
Hexamethylphosphoramide (HMPA) has not been included in the correlation, since it causes an unexpectedly high bathochromic shift of the UV/vis absorption maximum.

Since, in P1, the $\mathrm{N}-\mathrm{H}$ function is substituted, the effect of HBA-ability ( $b$ ) should be decreased, but this was not observed. The remaining influence of the HBA-ability of the solvent on the polymeric dye might be ascribed to an $\mathrm{N}-\mathrm{H}$ end group, for example. Especially for short-chain polymers a visible influence of end groups is conceivable. The influences of $\mathrm{H}$-bonding $(a+b)$ are almost unchanged. The most dominant effect on the solvatochromic behavior is caused by interaction with solvents of different dipolarity/polarizability which is reflected in the large coefficient $s$ in eqn (1) or coefficients $d$ and $e$ in eqn (2). ${ }^{8 d}$ The dipolar structures of dyes with an enlarged $\pi$-system usually are stabilized within the molecule rather than by interaction with a solvent molecule. To our surprise, the effect of polarizability and dipolarity of P1 increases significantly compared with M1 (the rate $\mathrm{SP} / \mathrm{SdP}$ remains unchanged). This could firstly be ascribed to the fact that $\mathbf{P 1}$ exhibits poor solubility in some solvents, if the chain length gets larger. In those solvents only very short chains, probably dimers or trimers, are measured by UV/vis spectroscopy. Secondly, the presence of $\mathrm{N}-\mathrm{H}$ end groups found by the unchanged high influence of SB, speaks for such short chains. The solvatochromic shift of P1 from $n$-hexane to HMPA is $3140 \mathrm{~cm}^{-1}$, and from $n$-hexane to DMSO is $2400 \mathrm{~cm}^{-1}$, which are much larger than that of M1.

For P2a, the influence of HBA-solvents is smaller, since the $\mathrm{N}-\mathrm{H}$-functionality is not present anymore. The residual effect may point to an $\mathrm{N}-\mathrm{H}$-end group again. The influence of polarizability and dipolarity remains unchanged compared with M1. The solvatochromic shift is decreased compared with those of M1 and P1a. From $n$-hexane to HMPA it is $1200 \mathrm{~cm}^{-1}$, and from $n$-hexane to DMSO it is $1180 \mathrm{~cm}^{-1}$.

\section{Conclusions}

In this article, the deep blue monomer M1 and four $\pi$-conjugated polymers based on aminobenzodione are described. M1 is very soluble in common organic solvents and suitable for Buchwald amination and Stille and Suzuki coupling because of the bromine and alkylamino substituent groups. The polymers exhibit quite low bandgaps (1.08-1.47 eV), high photostability and a large solvatochromic shift up to $3140 \mathrm{~cm}^{-1}$. Furthermore, the polymers show broad UV/vis absorption bands in a range from 400 to $900 \mathrm{~nm}$ with high extinction coefficients of 1.7 to 
$3.1 \times 10^{4} \mathrm{~L} \mathrm{~mol}^{-1} \mathrm{~cm}^{-1}$. This matches well with the solar photon most intense flux. The broad absorption in the visible region of the spectra, combined with high color depth, high photostability and a low bandgap render aminobenzodionebased polymers interesting as building blocks for optoelectronic materials.

\section{Acknowledgements}

Financial support from BASF Schweiz AG, Basle, Switzerland, China Scholarship Council (CSC) and German Academic Exchange Service (DAAD) is gratefully acknowledged. Dr P. Hayoz from BASF Schweiz AG, Basle is kindly thanked for helpful discussions.

\section{References}

1 (a) T. Limori, T. Naito and N. Ohta, J. Phys. Chem. C, 2009, 113, 4654-4661; (b) H. Kim, W. A. Goddard, S. S. Jang, W. R. Dichtel, J. R. Heath and J. F. Stoddart, J. Phys. Chem. A, 2009, 113, 2136-2143.

2 (a) S. R. Marder, B. Kippelen, A. K.-Y. Jen and N. Peyghambarian, Nature, 1997, 388, 845-851; (b) C.-G. Liu, W. Guan, P. Song, L.-K. Yan and Z.-M. Su, Inorg. Chem., 2009, 48, 6548-6554.

3 (a) Photoinduced Electron Transfer, ed. M. A. Fox and M. Chanon, Elsevier, Amsterdam, 1998; (b) H. Kurreck and M. Huber, Angew. Chem., 1995, 107, 927-947.

4 (a) K. Zhang and B. Tieke, Macromolecules, 2011, 44, 45964599; (b) P. A. Ulmann, H. Tanaka, Y. Matsuo, Z. Xiao, I. Soga and E. Nakamura, Phys. Chem. Chem. Phys., 2011, 13, 21045-21049; (c) K. Zhang, B. Tieke, J. C. Forgie, F. Vilela and P. J. Skabara, Macromolecules, 2012, 45, 743750; (d) H. Zhang and B. Tieke, Polym. Chem., 2014, 5, 646652.

5 (a) W. Cui, J. Yuen and F. Wudl, Macromolecules, 2011, 44, 7869-7873; (b) P. Deng, L. Liu, S. Ren, H. Li and Q. Zhang, Chem. Commun., 2012, 48, 6960-6962; (c) W. Hong, C. Guo, Y. Li, Y. Zheng, C. Huang, S. Lu and A. Facchetti, J. Mater. Chem., 2012, 22, 22282-22289.

6 (a) O. Annen, R. Egli, R. Hasler, B. Henzi, H. Jakob and P. Matzinger, Rev. Prog. Color. Relat. Top., 1987, 17, 72-85; (b) C. W. Greenhalgh, J. L. Carey and D. F. Newton, Dyes Pigm., 1980, 1, 103-120.

7 (a) C. W. Greenhalgh, J. L. Carey and D. F. Newton, J. Soc. Dyers Colour., 1994, 110, 178-184; (b) S. J. Bentley and D. J. Milner, Synth. Commun., 1996, 26, 95-100; (c) Z. Cai, J. Gao, X. Li and B. Xiang, Opt. Commun., 2007, 272, 503508; (d) G. Hallas and C. Yoon, Dyes Pigm., 2001, 48, 107-119. 8 (a) A. A. Gorman, M. G. Huthings and P. D. Wood, J. Am. Chem. Soc., 1996, 118, 8497-8498; (b) S. Spange, S. Prause, E. Vilsmeier and W. R. Thiel, J. Phys. Chem. B, 2005, 109, 7280-7289; (c) A. Oehlke, K. Hofmann and S. Spange, New J. Chem., 2006, 30, 533-536; (d) K. Hofmann, S. Brumm, C. Mende, K. Nagel, A. Seifert, I. Roth, D. Schaarschmidt, H. Lang and S. Spange, New J. Chem., 2012, 36, 1655.

9 D. J. Zillman, G. C. Hincapie, M. R. Savari, F. G. Mizori and

T. E. Cole, Tetrahedron Lett., 2010, 51, 3033-3036.

10 D. S. Surry and S. L. Buchwald, Chem. Sci., 2011, 2, 27-50.

11 P. K. Nayak, G. G. Belmonte, A. Kahn, J. Bisquert and D. Cahen, Energy Environ. Sci., 2012, 5, 6022-6039.

12 H. Zhang, I. Welterlich, J.-M. Neudoerfl, B. Tieke, C. Yang, X. Chen and W. Yang, Polym. Chem., 2013, 4, 4682-4689.

13 H. Zhang, J.-M. Neudoerfl and B. Tieke, Macromolecules, 2013, 46, 5842-5849.

14 T. Beyerlein and B. Tieke, Macromol. Rapid Commun., 2000, 21, 182-189.

15 I. Welterlich and B. Tieke, Polym. Chem., 2013, 4, 3755-3764.

16 (a) C. Reichardt, Chem. Rev., 1994, 94, 2319-2385; (b) P. Mueller, Pure Appl. Chem., 1993, 22, 409-416.

17 (a) M. J. Kamlet, T. N. Hall, J. N. Hall, J. Boykin and R. Taft, J. Org. Chem., 1979, 44, 2599-2604; (b) R. W. Taft and M. J. Kamlet, J. Am. Chem. Soc., 1976, 98, 2866-2894; (c) M. J. Kamlet and R. W. Taft, J. Am. Chem. Soc., 1976, 98, 377-383.

18 (a) J. Catalán and C. Díaz, Liebigs Ann., 1997, 1941-1949; (b) J. Catalán and C. Díaz, Eur. J. Org. Chem., 1999, 885-891; (c) J. Catalán, V. López and P. Pérez, Liebigs Ann., 1995, 793795.

19 (a) J. Catalán, C. Díaz, V. López, P. Pérez, J.-L. G. d. Paz and J. G. Rodríguez, Liebigs Ann., 1996, 1785-1794; (b) J. Catalán, J. Palomar, C. Díaz and J.-L. G. d. Paz, J. Phys. Chem. A, 1997, 101, 5183-5189.

20 J. Catalán and H. Hopf, Eur. J. Org. Chem., 2004, 4694-4702. 21 J. Catalán, J. Phys. Chem. B, 2009, 113, 5951-5960. 